\title{
MEDICINE AND SURGERY.
}

Vol. XIII.

JULY, 1824.

No. TII.

Observations on the Functions of the Absorbent System. By Jonathan Knight, Professor of Anatomy and Physiology in Yale College.

7.FFORE the discovery of the Lymphatic system of vessels, all 1 is absorption, both of solids and fluids, was supposed to be atured on by the veins. Since their discovery, they have been coubidered by most physiologists, as the sole agents of this process. In most of the late systems of Physiology, this doctrine is laid down in the broallest terms, and is considered as settled. What the object of this system of vessels is to take up and convey to the blood, various substances, either for the purposes of nutrition, or for the removal of useless parts out of the system, appears to be unquestionable; but I have long doubted their performance of the extensive operations which have been assigned to them. The subject is involved in olsscurity and where we find such men as Haller, Meckel, Hunter and Bichât in doubt upon it, others may well proceed with much caution.

I shall attempt to shew that the object of this system of vessels is to absorb and convey to the blood, those substances, (and which : are principally and perhaps, solely fluids.) only, which are found in the $\%$ wities of the body; including under the term cavities, those? which having no external conimunication, are lined by a berous membrane; those which having an external communicaThon, are lined by a mucous membrane, and all the cells of the 1liblar membrane. This idea excludes the lymphatics from all (4) 29 
agency in that gradual and constant removal of the solids, w: ich is continually going on in the living body, and almost of necessity, attributes this removal to the action of the veins.

The negative part of the proposition, that these vesselghave no agency in the removal of the solid parts of the body, all that is necessary to be proved, and to this; I shall princtpally confine my remarks.

That this opinion is true, I argne, in the first place, from the absence of all direct proof of their agency in this process. The idea that they are the sole agents of absorption has rnther been taken for granted, than proved. The object of the first discoverers of these vessels, and their followers, has bcen to prove, either, merely that they were absorbents, or that they were the sole aborbents of particular substances, as the chyle, \&c. In this they appear to have succeeded, but so far as I know, no direct proof of their agency in the removal of the solids has been offered. Hunter attempted to prove, and in the opinion of many succeeded, that venous absorption docs not take place from the surface of the intestines. 'This proves only that the lacteals are the sole absorbents from the intestinal cavity. For aught that appears from these experiments, the veins may be the agents which effect the gradual removal of the substance of the intestines themselves; or that they are what Bichat calls the nutritive absorbents of the part. Oiher physiologists have provêd, or attempted to prove, the same thing with regard to the removal of fluids from the surfaces of other organs; but all are deficient in any direct proof of the agency of the absorbents in the removal of the organs themselves. This has been rather a matter of inference than of proof. It is nothing new for discoverers to at: tribute more to the objects discovered, than subsequent examinations confirm; nor for an crror thus introduced to be exlensively believed. 'Thus when this set of vessels was discovered, and when it was proved that they were atsorbents, it is not \&range that they should, upon very slight grounds, be regarded as the sole agents of this process.

$2 \mathrm{dly}$, It is strongly probable, if not certain, that the veins do absorb a portion of the solids. It can scarcely be questioned that the carbon of the body is tiken up by the minute veins in every part of the body, and is eliminated from the system, pirincipally, at the lungs. That the carbon is taken up directly by the capillary veins, is inferred from the change which takes place in the colour and properties of the blood while passing through them, or while passing from the capillary arteries to the capillary veins. This change is exactly the converse of that which takees place in this fluid in the lungs. In the lungs it is caused bisthen 
abstraction of carbon from the blond; it is therefore reasonable to infer that the alteration which takes place in the capillaries of the system, is effected by the addition to it of this substance. In the lungs the blood is changed from a dark to a bright scarlet colourr, by the abstraction of carbon : in the extreme vessels the change is from scarlet to black, by the addition, or absorption of the same substance. The experiments of Migendie and Delisle might here also be mentioned, by which they attempted to prove the conveyance of poisonous substances into the system through the medium of the blood vessels. I have, however too little confidence in a set of severe experiments upon the living body, instituted to prove a physiological point, to insist much upon them. The experimenters commonly succeed in proving their position, at leas! to their own satisfaction. Whether this results from an inaccurate observation of the phenomena, as theypccur, or whether, from the violence done to the animal in the cousse of the experiment, the ordinary functions of the several parts are not properly performed, it is unnecessary to determine. The fact, that very various and entirely opposite opinions concerning the action of many parts of the animal body have been apparently proved by different experimenters, is sufficient to put us upon our guard in giving them credit. It is said also, that these experiments of Magendie, have been repeated by others with a very different result.

If then the veins do absorb a portion of the solid matter of the body, the fair inference is that they may and probably do absorb the whole.

$3 \mathrm{dly}$, One portion of this system of vessels, viz. the lacteals, has always been believed to be confined in its action, to fluids within a cavity. These vessels have never been supposed to absorb any other substanse than the chyle or fluid formed by

- digestion in the cavity of the alimentary canal. The lacteals are an important part of the general system of the absorbents. In structure thcy are the same, though commencing differently, they finally unite in a common trunk. 'They sympathize strongly with each other, both in health and disease. In vessels so similar in structure, and in so many ways connected with cach other, the fair inference is, that they are alike in function, and that, as the lacteals take up only fluids from a cavity, the same is true of the lymphatics.

4thly, The diseases which arise from a torpid state of the minute branches of the lymphatics, or from pressure upon their trunks, affect only the cavities of the body. Thus dropsies of the large cavities, or of the cellular membrane, often arise from. sach of these causes. But whoever heard of an enlargement 
of the muscles, bones, or of any of the solid parts of the body, having been produced by either of them? This surely ought sornetimes to occur if the lymphatics are the sole absorbents of it both solids and fluids.

5thly, The situation of the lymphatics is in favour of the opinion that their action is confined to fluids within cavities. They are found aburdantly in the subcutaneous and other cellular membrane, and upon the surfaces of all the cavities, while comparatively few exist in the solid parts of the body. So abundant are they in the serous inembranes, that these have been considered by many, as little more than a tissue of exhalant and lymphatic vessels. It is no objection to this argument, that lymphatics have been observed, less abundantly, in every part of the body; for no one will pretend that they are more generally dilfusedy the cellular nembrane.

6 thly, 'The analysis of ly mph confirms the opinion advanced. The following is the account given by $W$. Brande of the composition of this fluid. (Philosophical Magazine for Aug. 1812 and Eclectic Repertory, vol. 3. p. 298. "The fluid found in the thoracic duct of animals that have been kept for twenty-four hours without food, is perfectly transparent and colourless, and seems to differ in no respect from that which is contained in the lymphatic vessels.

-It has the following properties:

'1. It is miscible in every proportion with water.

6. It produces no change in vegetable colours.

'3. It is neither coagulated by heat nor acids, nor alcohol, but is generally rendered slightly turbid by the last reagent.

- 4. When evaporated to dryness, the residuum is very small in quantity, and slightly affects the colour of violet paper, changing it to green.

' 5 . By incineration in a platina crucible, the residuum io found to contain a minute portion of muriate of soda; but I conld not discover in it the slightest indications of iron.

6. In the examination of this fluid, I availed myself with some advantage of those modes of electro-chemical analysis, which on a former occasion 1 have described to this Society.' H

'When the lymph was submitted to the electrical action of a battery consisting of twenty pairs of four-inch plates of copper and zinc, there was an evolufion of alkaline matter at the negative surface, and portions of coagulated albumen were separated. As far as the small quantities on which I operated enabled me to ascertain, muriatic acid only was evolved at the positive surface.'

* Phil. Trans. 1804. p. 373. 


\section{1}

This analysis I have given entire in the words of the author. From this it will be seen that all that exists in pure lymph, basides water, which makes up the principal part of it, is albumen and muriate of soda. These substances exist in most, if not all the animal fluids, and in the simplest of them, such as serum, \&c. no others are usually found. Indeed the strong resemblance, if not identity, between the lymph, and the fluid found in all the closed cavities cannot fail to strike the mind of the enquirer.

We find in the lymph no indications of the presence of the matter of the muscles, bones, or of any other of the great variety of the solid portions of the body, either in their proximate or ultimate forms. This is wholly inexplicable upon the idea that the lymphatics are the sole absorbents of the body, or indeed that they are at all concerned in the removal of its solid portions.

1 have endeavoured to present this subject in the most brief and simple munner, not embarrassing it with extraneous matter, nor noticing many of the ingenious opinions which have been advanced within these few years, upon various parts of the general subject of absorption. My sole object has been to shew, that the absorbents are confined in their action, to fluids within cavities.

Observations on the various methods of treating the fractures of the Os Femoris, with Cases, in which a new apparatus was successfully used. By LUKe Howz, M.D. ,

[Communicated for the New England Journal of Medicius and Surgery.]

THIS part of surgery has, undnubtedly. received that full share. of attention, both from allcient and modern surgeons, which the importance of the subject demanded. It was conceded by Celsus, that, in oblique fractures of the femur, a shortening would, inevitably, take place. And Benjamin Bell, lamenting, that the modes of treatment in use, would sometimis fail, says, "lindeed: an effectual method of securing very oblique fracture in any of the bones of the extremities, and especially the thigh bone, is perhaps one of the greatest desiderata in modern surgeny.' The ancients merited much for ingenuity in their various machinery for the extension of a fractured femur; and their frequent failures of success are, principally, to be imputed to their very imperfect knowledge of the indications of treatment. Later surgeons have been better informed in respect to the causes, which so. often defeat their ikill ; and have directed their attention to the source of the evil with better prospects of success.

We, still, too: often, pitness limbs shortened and distorted by 